\title{
Iterative Krylov Methods for Acoustic Problems on Graphics Processing Unit
}

\author{
Abal-Kassim Cheik Ahamed* $\quad$ Frédéric Magoulès ${ }^{\dagger}$
}

\begin{abstract}
This paper deals with linear algebra operations on Graphics Processing Unit (GPU) with complex number arithmetic using double precision. An analysis of their uses within iterative Krylov methods is presented to solve acoustic problems. Numerical experiments performed on a set of acoustic matrices arising from the modelisation of acoustic phenomena inside a car compartment are collected, and outline the performance, robustness and effectiveness of our algorithms, with a speed-up up to $28 \mathrm{x}$ for dot product, $9.8 \mathrm{x}$ for sparse matrix-vector product and solvers.
\end{abstract}

Keywords: Linear algebra; Iterative Krylov methods; CSR matrix; GPU; CUDA; Acoustic; Helmholtz equation; Parallel computing

\section{Introduction}

Linear algebra analysis has always been extremely useful when solving partial differential equations arising from many domains such as physics and biology models. Even though Graphics Processing Units (GPUs) were first designed for graphic applications, they also represent a high potential for scientific computing and its applications to both physics and engineering. General-Purpose GPUs allow the developers to harness the high computational power of graphics cards to accelerate general-purpose scientific and engineering computing. The peak performance of CPUs and GPUs is significanlty different, due to the inherently different architectures between these processors. In this work we focus on Compute Unified Device Architecture (CUDA) [45], proposed by NVIDIA in 2006, an appropriate and suitable language for NVIDIA graphics card. CUDA has offered a new vision in high performance computing. In this paper, we analyse double precision complex number arithmetics algorithms of Alinea [11, 10], our own research group library, which proposes linear algebra operations and iterative Krylov on both CPU and GPU clusters for real and complex number arithmetics in single and double precision.

The acoustic problem is steered in the frequency domain by the Helmholtz equation with suitable boundary conditions. The matrix of the linear system arising from the finite element discretization of the acoustic problem has a very huge size on high frequency regime. Several discretization techniques like infinite element [3, 2, 1] or stabilized finite element [21] allows to reduce the size of the matrix. The problem to solve comes from the discretization of the Helmholtz equation in a bounded domain $\Omega$, with outside boundary $\Gamma=\partial \Omega$. The Helmholtz equation is formulated as: $-\nabla^{2} u-k^{2} u=g$, where $k=\frac{2 \pi F}{c}$ is the wavenumber of the frequency $F \in \mathbb{R}$ and $c \in \mathbb{R}$ is the velocity of the medium, which is different in space. In this work, we consider Dirichlet boundary conditions along a part of $\Gamma$. Numerical experiments done on a set of acoustic finite element matrices are exhibited and show the performance, robustness and accuracy of linear algebra operations and their uses within iterative Krylov methods for solving acoustic problem modeled by Helmholtz equation.

\footnotetext{
* CUDA Research Center, Applied Mathematics and Systems Laboratory, École Centrale Paris, France.

$\dagger$ CUDA Research Center, Applied Mathematics and Systems Laboratory, École Centrale Paris, France (correspondence, frederic.magoules@hotmail.com).
} 
The plan of this paper is the following. Section 2 presents the industrial test cases involved to analyze our algorithms. Section 3 presents numerical results of linear algebra operations required to carry out iterative Krylov methods such as addition of vectors, scale of vectors, sparse matrix-vector multiplication (SpMV), etc. Section 4 gives numerical tests on iterative Krylov methods, and Section 5 gives conclusion.

\section{Application: automotive acoustic}

This part of the paper gives the main features of the finite element meshes used associated with the acoustic problems arising from the automotive industry [34], namely car compartments: Audi (Audi3D) and Twingo (Twingo3D). The car compartment problem is representative of acoustics cavity. Fig. 1 illustrates respectively the Audi3D and Twingo3D mesh for a given mesh size $(h)$.
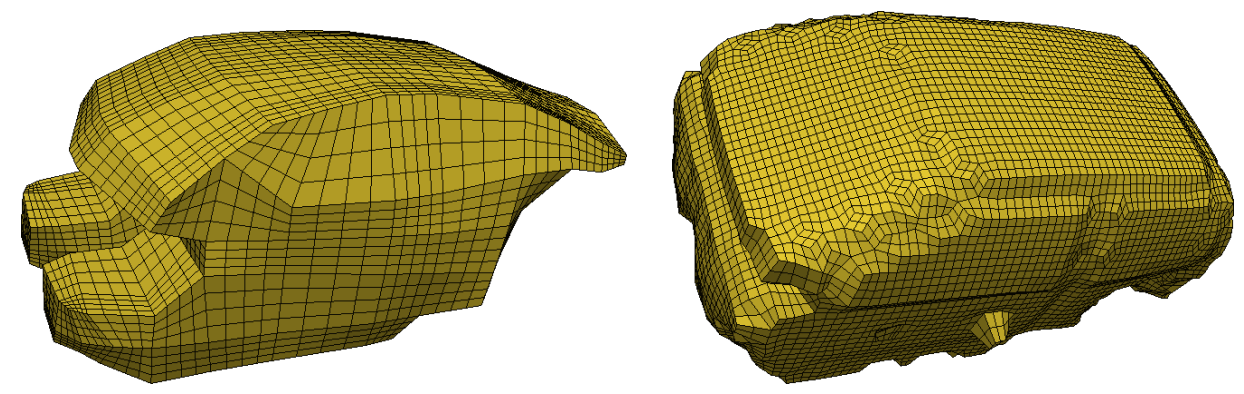

Figure 1: Audi (Audi3D) and Twingo (Twingo3D)

The matrices used to analyze and evaluate our algorithms are obtained from the finite element discretization of the acoustic problem, governed by the Helmholtz equation. The matrices are sparse large size, i.e., most values are zero. In this way, Compressed Sparse Row (CSR) [6], is considered to store these matrices. Table 1 reports the matrices associated with the meshes of the car compartments. These features and characteristics are given in the third column. The sparse structure pattern and an histogramm of the distribution of nonzero values are respectively given in the first and second column.

The numerical experiments have been carried out on a workstation based on an Intel Core i7 920 2.67Ghz, which has 4 physical cores and 4 logical cores, 12GB RAM, and two NVIDIA graphics card: a Tesla K20c (device \#0) with 4799GB memory and GeForce GTX 570 with $1279 \mathrm{MB}$ memory (device $\# 1$ ). The cards are double precision compatible. In the following Tesla K20c and GTX 570 will be denoted respectively gpu\#0 and gpu\#1. For the sake of accuracy, we perform each operation 100 times, and the time indicated corresponds to the average time.

\section{$3 \quad$ Linear Algebra Operations}

This section introduces linear algebra algorithms such as assign of a vector, scale of vectors, element wise product, addition of vectors, dot product and sparse matrix-vector products. CUDA was originally dedicated for integer arithmetics and then for real numbers arithmetics, with a decreasing of performance of computations. Since, a complex number is a set of two real numbers composed of real and imaginary part, implementation is feasible by designing a structure of two real numbers. CUDA library includes a structure called cuComplex, but for performance reasons, we specify our own complex class template structure complex $<\mathrm{T}>$ that offers all the operations given by the standard std: : complex. As a result, in order to get the most benefits of GPU architecture, the elementary linear operation kernel requires to be reimplemented [22, 6, 5]. In reference [10], an analysis carried out on real number artihmetics 
Table 1: Sketches of Audi, Twingo FE matrices

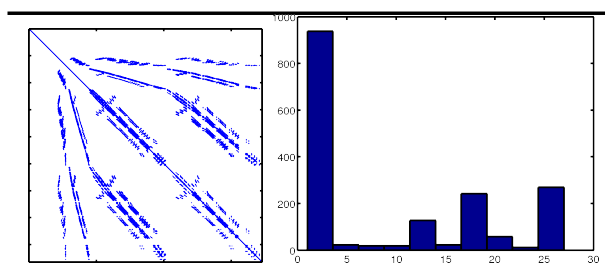

\section{Audi3D-1}

$\mathrm{h}=$ size $=1,727 \quad$ density $=0.550$

bandwidth $=1,436 \quad$ nonzero $=16,393$

nonzero $/ \mathrm{h}=9.492 \quad \max$ row $=27$ nonzero $/ \mathrm{h}$ stddev $=10.205$

3D acoustic FE matrix. Audi car (mesh size =0.133425, length wave $=3.5$ ).

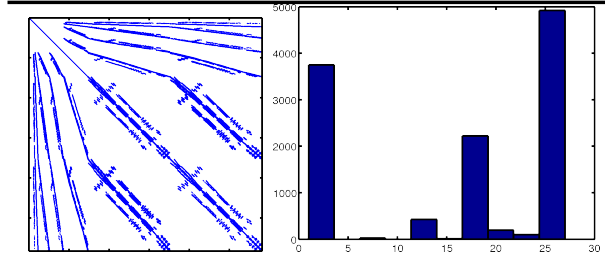

Audi3D-2

3D acoustic FE matrix. Audi car (mesh size $=0.066604$, length wave $=3.5$ ).

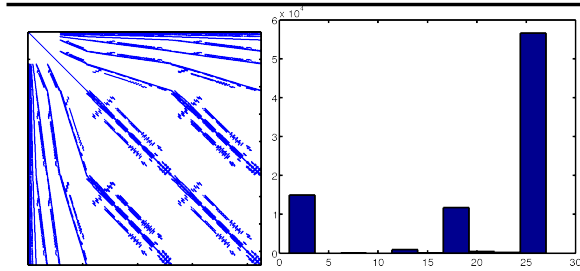

Audi3D-3

3D acoustic FE matrix. Audi car (mesh size $=0.033289$, length wave $=3.5$ ).

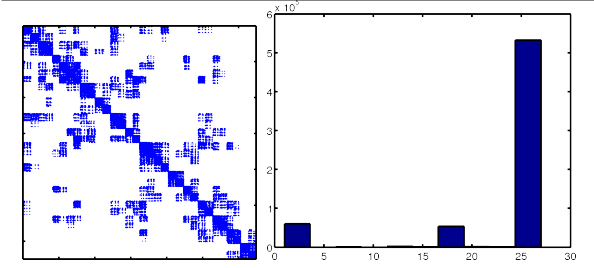

Audi3D-4

$\mathrm{h}=$ size $=85,001 \quad$ density $=0.025$

bandwidth $=84,474 \quad$ nonzero $=1,781,707$

nonzero $/ \mathrm{h}=20.961 \quad \max$ row $=27$ nonzero $/$ h stddev $=9.832$

3D acoustic FE matrix. Audi car (mesh size $=0.016643$, length wave $=3.5$ ).

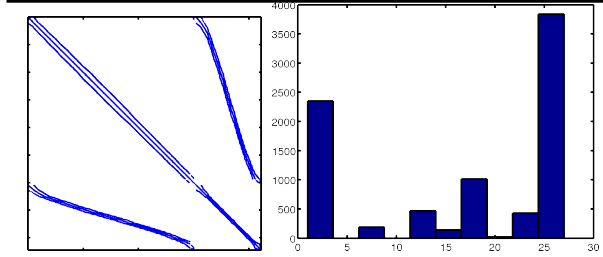

Twingo3D-0

$\mathrm{h}=$ size $=648,849 \quad$ density $=0.004$

bandwidth $=520,461 \quad$ nonzero $=15,444,211$

nonzero $/ \mathrm{h}=23.802 \quad \max$ row $=27$

nonzero $/ \mathrm{h}$ stddev $=7.720$

3D acoustic FE matrix. Twingo car (mesh size $=0.077866$, length wave $=9.5$ ).

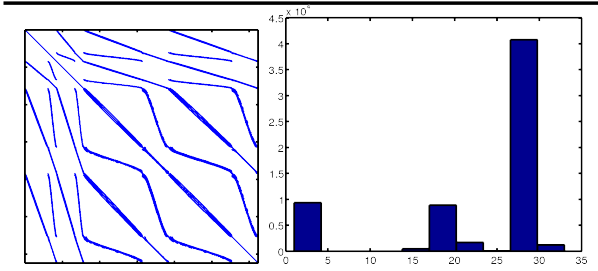

Twingo3D-1

$\mathrm{h}=$ size $=8,439 \quad$ density $=0.202$

bandwidth $=6,268 \quad$ nonzero $=143,889$

nonzero $/ \mathrm{h}=17.050 \quad \max$ row $=27$

nonzero $/ \mathrm{h}$ stddev $=11.047$

3D acoustic FE matrix. Twingo car (mesh size $=0.038791$, length wave $=9.5$ ).

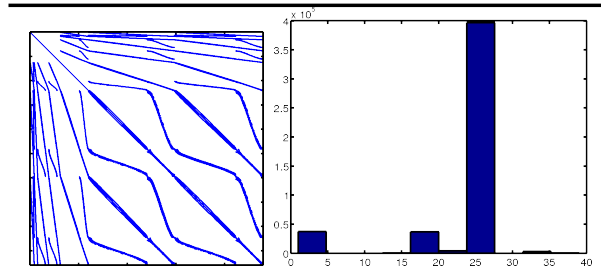

Twingo3D-2

$\mathrm{h}=$ size $=62,357 \quad$ density $=0.035$

bandwidth $=53,935 \quad$ nonzero $=1,351,521$

nonzero $/ \mathrm{h}=21.674 \quad \max$ row $=33$

nonzero $/$ h stddev $=9.364$

3D acoustic FE matrix. Twingo car (mesh size $=0.019379$, length wave $=9.5$ ). 
with double precision with a suitable implementation of the CUDA kernel presents excellent speed-up for linear algebra operations and iterative Krylov methods [11]. The finite element discretization of the Helmholtz equation for acoustic problems conducts to complex number arithmetics matrices. In this paper, we give an extension of this analysis with acoustic problem. We develop efficient iterative Krylov methods for solving linear systems with complex number arithmetics. As proved in [12] for real number arithmetics, our template code gives effective results compared to Cusp [7], CUBLAS [43], CUSPARSE [44]. But performance for complex number arithmetics with double precision remains a defiance, and dynamic auto-tuning of the GPU grid should be considered considered [10].

The complex double precision running times in milliseconds (ms) of the assign operation are collected in Table 2, with $h$ the size of the vector.

Table 2: Assign of vector (ZASSIGN)

\begin{tabular}{cccccccccc}
\hline $\mathbf{h}$ & $\mathbf{c p u}$ & $\mathbf{c p u}$ & $\mathbf{g p u} \# \mathbf{0}$ & gpu\#0 & gpu\#1 & gpu\#1 ratio\#0 ratio\#1 \\
& time $(m s)$ & Gflops & time $(m s)$ & Gflops & time $(m s)$ & Gflops & cpu/\#0 & cpu/\#1 \\
\hline 648,849 & 1.10 & 0.59 & 0.16 & 4.06 & 0.20 & 3.27 & $\mathbf{6 . 8 8}$ & $\mathbf{5 . 5 4}$ \\
$2,000,000$ & 4.00 & 0.50 & 0.41 & 4.92 & 0.46 & 4.36 & $\mathbf{9 . 8 4}$ & $\mathbf{8 . 7 2}$ \\
$9,000,000$ & 18.33 & 0.49 & 1.79 & 5.04 & 1.69 & 5.31 & $\mathbf{1 0 . 2 7}$ & $\mathbf{1 0 . 8 2}$ \\
$14,000,000$ & 27.50 & 0.51 & 2.63 & 5.32 & 2.86 & 4.90 & $\mathbf{1 0 . 4 5}$ & $\mathbf{9 . 6 3}$ \\
\hline
\end{tabular}

In the following, all kernels compute the global index of each thread as follows:

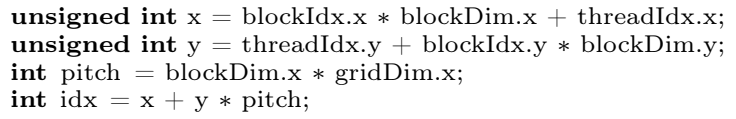

The scale scale operation kernel is described as follows

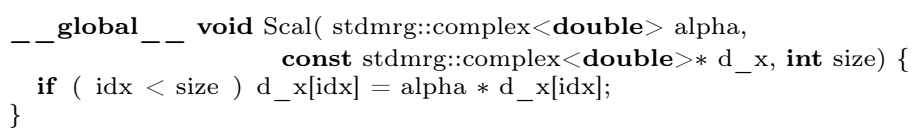

In Table 3, we collect the execution times of the scale scale operation.

Table 3: Scale of vectors (ZSCAL)

\begin{tabular}{ccccccccc}
\hline $\mathbf{h}$ & $\mathbf{c p u}$ & $\mathbf{c p u}$ & gpu\#0 & gpu\#0 & gpu\#1 & gpu\#1 & ratio\#0 & ratio\#1 \\
& time $(m s)$ & Gflops & time $(m s)$ & Gflops & time $(m s)$ & Gflops & cpu/\#0 & cpu/\#1 \\
\hline 648,849 & 5.56 & 0.70 & 0.20 & 19.47 & 0.21 & 18.34 & $\mathbf{2 7 . 7 8}$ & $\mathbf{2 6 . 1 7}$ \\
$2,000,000$ & 15.71 & 0.76 & 0.46 & 26.04 & 0.53 & 22.56 & $\mathbf{3 4 . 1 0}$ & $\mathbf{2 9 . 5 4}$ \\
$9,000,000$ & 80.00 & 0.68 & 1.92 & 28.08 & 2.33 & 23.22 & $\mathbf{4 1 . 6 0}$ & $\mathbf{3 4 . 4 0}$ \\
$14,000,000$ & 120.00 & 0.70 & 2.94 & 28.56 & 3.57 & 23.52 & $\mathbf{4 0 . 8 0}$ & $\mathbf{3 3 . 6 0}$ \\
\hline
\end{tabular}

Double-precision complex Alpha $X$ Plus $Y$ (Zaxpy), i.e., $y[i]=\alpha \times x[i]+y[i]$, is a level one (vector) operation between two complex number arithmetics vectors in the Basic Linear Algebra Subprograms (BLAS) package. The simple CUDA kernel of Zaxpy is implemented as follows:

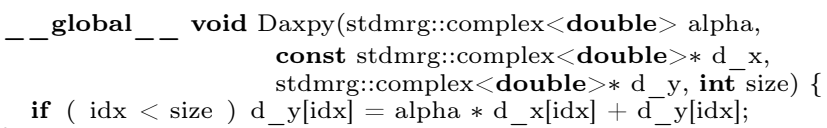

In Table 4, we present the complex number arithmetics with double precision execution times in milliseconds (ms) of Zaxpy operation. 
Table 4: Addition of vectors (ZAXPY)

\begin{tabular}{|c|c|c|c|c|c|c|c|c|}
\hline $\mathbf{h}$ & $\begin{array}{c}\mathbf{c p u} \\
\text { time (ms) }\end{array}$ & $\begin{array}{c}\text { cpu } \\
\text { Gflops }\end{array}$ & $\begin{array}{c}\mathbf{g p u} \# \mathbf{0} \\
\text { time (ms) }\end{array}$ & $\begin{array}{c}\text { gpu\#0 } \\
\text { Gflops }\end{array}$ & $\begin{array}{c}\mathbf{g p u} \# \mathbf{1} \\
\text { time (ms) }\end{array}$ & $\begin{array}{c}\text { gpu\#1 } \\
\text { Gflops }\end{array}$ & $\begin{array}{c}\operatorname{ratio} \# \mathbf{0} \\
c p u / \# 0\end{array}$ & $\begin{array}{c}\operatorname{ratio} \# \mathbf{1} \\
c p u / \# 1\end{array}$ \\
\hline 648,849 & 5.56 & 0.93 & 0.26 & 20.04 & 0.27 & 19.52 & 21.44 & 20.89 \\
\hline $2,000,000$ & 16.67 & 0.96 & 0.69 & 23.20 & 0.81 & 19.68 & 24.17 & 20.50 \\
\hline $9,000,000$ & 75.00 & 0.96 & 3.03 & 23.76 & 3.33 & 21.60 & 24.75 & 22.50 \\
\hline $14,000,000$ & 120.00 & 0.93 & 4.76 & 23.52 & 5.26 & 21.28 & 25.20 & 22.80 \\
\hline
\end{tabular}

The element wise product or element by element product, i.e., $y[i]=x[i] \times y[i]$. The CUDA kernel, is described simply as:

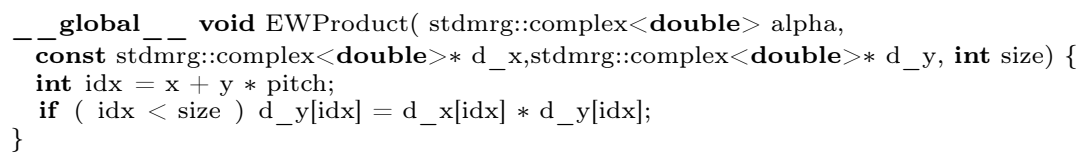

Table 5 exhibits the double precision execution times of the element by element product operation.

Table 5: Element wise product (ZAXMY)

\begin{tabular}{|c|c|c|c|c|c|c|c|c|}
\hline $\mathbf{h}$ & $\begin{array}{c}\text { cpu } \\
\text { time (ms) }\end{array}$ & $\begin{array}{c}\text { cpu } \\
\text { Gflops }\end{array}$ & $\begin{array}{c}\mathbf{g p u} \# \mathbf{0} \\
\text { time (ms) }\end{array}$ & $\begin{array}{c}\text { gpu\# } \\
\text { Gflops }\end{array}$ & $\begin{array}{c}\mathbf{g p u} \# \mathbf{1} \\
\text { time (ms) }\end{array}$ & $\begin{array}{c}\text { gpu\#1 } \\
\text { Gflops }\end{array}$ & $\begin{array}{c}\text { ratio } \# \mathbf{0} \\
c p u / \# 0\end{array}$ & $\begin{array}{c}\operatorname{ratio} \# \mathbf{1} \\
c p u / \# 1\end{array}$ \\
\hline 648,849 & 8.33 & 0.47 & 0.28 & 13.66 & 0.29 & 13.55 & 29.25 & 29.00 \\
\hline $2,000,000$ & 25.00 & 0.48 & 0.72 & 16.56 & 0.85 & 14.16 & 34.50 & 29.50 \\
\hline $9,000,000$ & 120.00 & 0.45 & 3.03 & 17.82 & 3.33 & 16.20 & 39.60 & 36.00 \\
\hline $14,000,000$ & 180.00 & 0.47 & 4.76 & 17.64 & 5.00 & 16.80 & 37.80 & 36.00 \\
\hline
\end{tabular}

Dot product operation can be very costly for large size vectors. Instead of performing a simple loop with simultaneous sums to compute the dot product, which is not very effective on GPUs, we perform it into two distinct tasks. The first is the element wise product of vectors and the second consists in summing all the results obtained at the first step. The reduction done at the second step associates each element of the input data with a thread, and at the end the partial sum of the $n^{\text {th }}$ first elements is stored in the first thread of the current block. The final dot product result is then computed as the sum of all the partial sums of the different blocks. The double precision execution times of the dot product on both CPU and GPU are exposed in Table 6 and Fig. 2. Table 7 gives the numerical results

Table 6: Dot product (ZDOT)

\begin{tabular}{ccccccccc}
\hline $\mathbf{h}$ & $\mathbf{c p u}$ & $\mathbf{c p u}$ & \multicolumn{2}{c}{ gpu\#0 } & gpu\#0 & gpu\#1 & \multicolumn{2}{c}{ gpu\#1 ratio\#0 ratio\#1 } \\
& time $(m s)$ & Gflops & time $(m s)$ & Gflops & time $(m s)$ & Gflops & cpu/\#0 & cpu/\#1 \\
\hline 648,849 & 5.56 & 0.93 & 0.33 & 15.83 & 0.33 & 15.94 & $\mathbf{1 6 . 9 4}$ & $\mathbf{1 7 . 0 6}$ \\
$2,000,000$ & 16.67 & 0.96 & 0.83 & 19.20 & 0.76 & 20.96 & $\mathbf{2 0 . 0 0}$ & $\mathbf{2 1 . 8 3}$ \\
$9,000,000$ & 80.00 & 0.90 & 3.23 & 22.32 & 3.23 & 22.32 & $\mathbf{2 4 . 8 0}$ & $\mathbf{2 4 . 8 0}$ \\
$14,000,000$ & 130.00 & 0.86 & 4.76 & 23.52 & 4.55 & 24.64 & $\mathbf{2 7 . 3 0}$ & $\mathbf{2 8 . 6 0}$ \\
\hline
\end{tabular}

of the norm operation.

As shown in Table 6 and Table 7, GPUs clearly show better results than CPU with complex number arithmetics in double precision. Much more than the dot product, the 

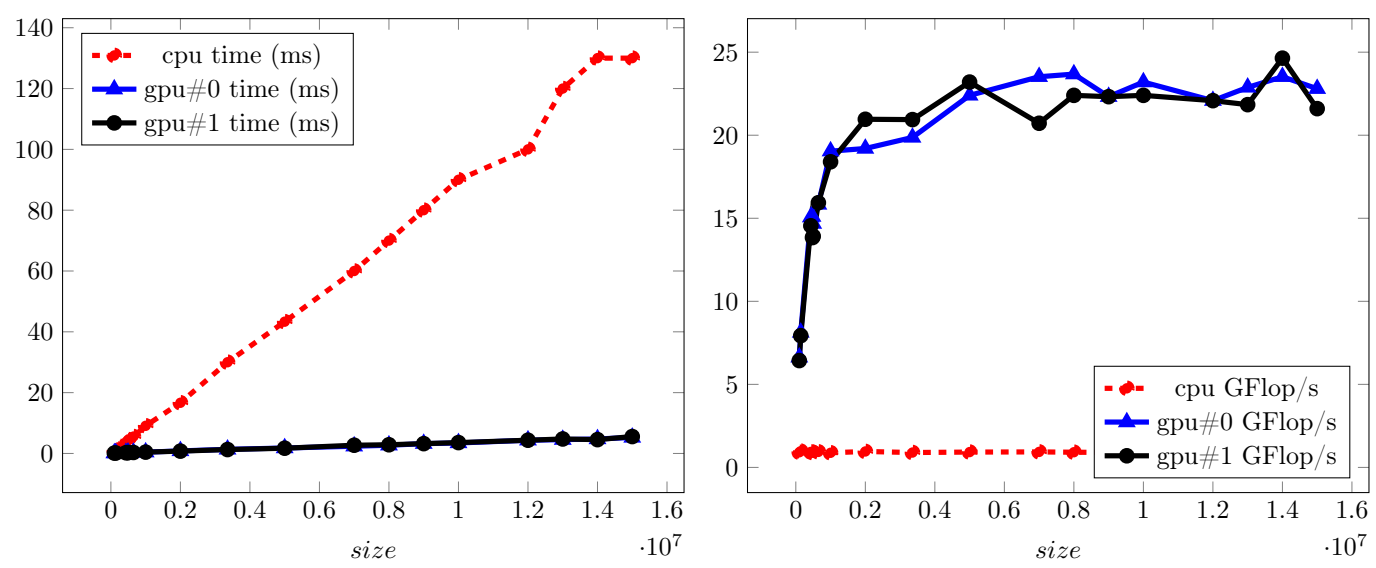

Figure 2: ZDOT [left: time in ms, right: GFlops]

Table 7: NormL2 (ZNORM)

\begin{tabular}{|c|c|c|c|c|c|c|c|c|}
\hline \multirow[t]{2}{*}{$\mathbf{h}$} & cpu & cpu & gpu $\# \mathbf{0}$ & gpu\#0 & & gpu $\# 1$ & ratio $\# 0$ & ratio $\# 1$ \\
\hline & time (ms) & Gflops & time (ms) & Gflops & time (ms) & Gflops & $c p u / \# 0$ & $c p u / \# 1$ \\
\hline 648,849 & 11.11 & 0.29 & 0.31 & 10.54 & 0.26 & 12.65 & 36.11 & 43.33 \\
\hline $2,000,000$ & 33.33 & 0.30 & 0.73 & 13.70 & 0.57 & 17.60 & 45.67 & 58.67 \\
\hline $9,000,000$ & 150.00 & 0.30 & 3.13 & 14.40 & 2.27 & 19.80 & 48.00 & 66.00 \\
\hline $14,000,000$ & 230.00 & 0.30 & 5.00 & 14.00 & 3.70 & 18.90 & 46.00 & 62.10 \\
\hline
\end{tabular}

SpMV is probably the most time consuming operation in sparse matrix computation. This is required on all Krylov iterative methods. As proved in [10], the performance of SpMV strongly depends on the properties of the matrix, particularly on the distribution of nonzero values. The following results are obtained with advanced auto-tuned techniques to organize threads on the CUDA grid. References [42, 52, 51, 23, 16] clearly showed the effectiveness of SpMV on GPU compared to CPU for real number arithmetics. The running time and the number of floating operations per second for SpMV with complex number arithmetics with double precision are reported in Table 8.

Table 8: SpMV CSR

\begin{tabular}{|c|c|c|c|c|c|c|c|c|}
\hline problem & $\begin{array}{c}\mathbf{c p u} \\
\text { time }(m s)\end{array}$ & $\begin{array}{c}\text { cpu } \\
\text { Gflops }\end{array}$ & $\begin{array}{c}\text { gpu\# } \# \mathbf{0} \\
\text { time (ms) }\end{array}$ & $\begin{array}{c}\text { gpu } \# \mathbf{0} \\
\text { Gflops }\end{array}$ & $\begin{array}{c}\mathbf{g p u} \# \mathbf{1} \\
\text { time (ms) }\end{array}$ & $\begin{array}{c}\text { gpu\#1 } \\
\text { Gflops }\end{array}$ & $\begin{array}{r}\operatorname{ratio} \# \mathbf{0} \\
c p u / \# 0\end{array}$ & $\begin{array}{c}\text { ratio } \# \mathbf{1} \\
c p u / \# 1\end{array}$ \\
\hline Audi3D-0 & 0.01 & 0.61 & 0.07 & 0.12 & 0.06 & 0.13 & 0.19 & 0.21 \\
\hline Audi3D-1 & 0.20 & 0.67 & 0.11 & 1.23 & 0.12 & 1.07 & 1.84 & 1.60 \\
\hline Audi3D-2 & 2.22 & 0.68 & 0.37 & 4.03 & 0.42 & 3.56 & 5.93 & 5.24 \\
\hline Audi3D-3 & 20.00 & 0.71 & 2.22 & 6.41 & 3.03 & 4.70 & 9.00 & 6.60 \\
\hline Audi3D-4 & 180.00 & 0.69 & 18.33 & 6.74 & 24.00 & 5.15 & 9.82 & 7.50 \\
\hline Twingo3D-0 & 1.67 & 0.69 & 0.28 & 4.06 & 0.33 & 3.45 & 5.88 & 5.00 \\
\hline Twingo3D-1 & 15.71 & 0.69 & 1.79 & 6.05 & 2.44 & 4.43 & 8.80 & 6.44 \\
\hline Twingo3D-2 & 140.00 & 0.66 & 14.29 & 6.51 & 16.67 & 5.58 & 9.80 & 8.40 \\
\hline
\end{tabular}




\section{Iterative Krylov methods}

After the analysis of linear algebra operations for complex number arithmetics with double precision, we now evaluate and analyze their uses within iterative Krylov methods [24, 42, 4, 52]. We have thus implemented a preconditionned bi-conjugate gradient stabilized method (P-Bi-CGSTAB), a preconditionned P-BiCGSTAB parametered (l) and a preconditionned transpose-free quasi-minimal residual method (P-tfQMR) [48], with optimized CUDA and dynamic auto-tuning on GPU. The data transfer between CPU and GPU consists of an important part of optimization [9] for optimal performance on GPGPU. In our Krylov methods codes, we take care to send once all required input data from CPU to GPU before beginning the iterations. Even so, at each computed dot product or norm, there is one copy back from GPU to CPU. Both CPU and GPU codes are strictly the same, but all linear algebra operations such as Zdot, Znorm, Zaxpy, or SpMV are performed on device (GPU) for the GPU version. The presented iterative Krylov methods are performed with a residual tolerance threshold of $1 \times 10^{-9}$, an initial guess of zero and 1000 maximum number of iterations. The numerical experiments presented in the following give an analysis of Krylov methods on CPU and GPU, with the same code, for complex number arithmetics with double precision. The CPU and GPU execution times and corresponding speed-up of Audi3D and Twingo3D are collected in Table 9 and Table 10. The results corroborate the effectiveness of

Table 9: Speed-up of Audi3D

\begin{tabular}{|c|c|c|c|c|}
\hline problem & $\#$ iter & CPU time $(\mathrm{s})$ & GPU time $(\mathrm{s})$ & speed-up \\
\hline \multicolumn{5}{|c|}{$P-B i C G S T A B$} \\
\hline Audi3D-1 & 21 & 0.01 & 0.030 & 0.33 \\
\hline Audi3D-2 & 53 & 0.24 & 0.106 & 2.26 \\
\hline Audi3D-3 & 94 & 4.01 & 0.703 & 5.71 \\
\hline Audi3D-4 & 183 & 85.70 & 9.209 & 9.31 \\
\hline \multicolumn{5}{|c|}{$P-B i C G S T A B(8)$} \\
\hline Audi3D-1 & 6 & 0.03 & 0.110 & 0.27 \\
\hline Audi3D-2 & 12 & 0.52 & 0.286 & 1.82 \\
\hline Audi3D-3 & 31 & 12.47 & 2.162 & 5.77 \\
\hline Audi3D-4 & 70 & 266.26 & 30.100 & 8.85 \\
\hline \multicolumn{5}{|l|}{$P-T F Q M R$} \\
\hline Audi3D-1 & 24 & 0.02 & 0.040 & 0.50 \\
\hline Audi3D-2 & 52 & 0.27 & 0.113 & 2.40 \\
\hline Audi3D-3 & 99 & 4.71 & 0.755 & 6.24 \\
\hline Audi3D-4 & 214 & 102.17 & 10.786 & 9.47 \\
\hline
\end{tabular}

Table 10: Speed-up of Twingo3D

\begin{tabular}{lcccc}
\hline problem & \#iter & CPU time (s) & GPU time (s) & speed-up \\
\hline$P$-BiCGSTAB & & & & \\
Twingo3D-0 & 563 & 1.85 & 1.008 & $\mathbf{1 . 8 4}$ \\
Twingo3D-1 & 1000 & 29.45 & 5.730 & $\mathbf{5 . 1 4}$ \\
Twingo3D-2 & 1000 & 295.66 & 37.670 & $\mathbf{7 . 8 5}$ \\
\hline $\begin{array}{l}\text { P-BiCGSTAB(8) } \\
\text { Twingo3D-0 }\end{array}$ & 1000 & 31.2 & 20.970 & \\
Twingo3D-1 & 1000 & 273.81 & 54.630 & $\mathbf{5 . 0 1}$ \\
Twingo3D-2 & 1000 & 2559.67 & 324.500 & $\mathbf{7 . 8 9}$ \\
\hline$P$-TFQMR & & & & \\
Twingo3D-0 & 366 & 1.34 & 0.626 & $\mathbf{2 . 1 4}$ \\
Twingo3D-1 & 954 & 30.4 & 5.438 & $\mathbf{5 . 5 9}$ \\
Twingo3D-2 & 1000 & 318.93 & 38.090 & $\mathbf{8 . 3 7}$ \\
\hline
\end{tabular}


GPU compared to CPU for solving sparse linear systems. The speed-up grows when the size of the problems increase for all tests, i.e., for a finer mesh GPU is more effective compared to CPU. For a finer mesh the assembled matrix turns into non appropriate size for memory of most of GPUs. To overcome this problem, one way consists in using domain decomposition method [49, 46, 50, 17, 36] based on iterative methods with interface conditions defined on the interface between the subdomains [29]. The Schwarz method [25, 26, 27, 8] is suitable for solving large size problem. To accelerate the convergence, many references [15, 20, 31, 30, 28] show the importance of these interface conditions. In order to implement this perspective for acoustic problems continuous optimized interface conditions between the subdomains must be implemented as in [19, 33, 32, 35]. Alternative discrete optimization techniques as introduced in [37, 47, 41, 38, 18, 40, 39] allow a fast and robust convergence of the Schwarz algorithm too. In [13, 14], the authors describe how domain decomposition method is effectively implemented on GPU and proved the robustness of Schwarz methods on a cluster of GPUs. The extension to the complex number arithmetics double precision, of the iterative Krylov methods, to solve the local subproblems defined in each subdomains, leads to similar speed-up. For the Audi car compartment, a speed-up up to $9.2 \mathrm{x}$ is obtained for eight subdomains.

\section{Conclusion}

In this paper we give an analysis of linear algebra operations together with their uses within iterative Krylov methods for solving acoustic problems on Graphics Processing Unit (GPU) with complex number arithmetics with double precision. Numerical tests have been carried out on two different system of accelerated generations of NVIDIA graphics card: GTX570 and Tesla K20c. A set of industrial matrices coming from the finite element discretization of acoustic problems modeled by the Helmholtz equation inside a car compartment are used to demonstrate the interest of using GPU device to perform linear algebra operations, and outline the robustness, performance and effectiveness of the proposed implementation.

\section{References}

[1] J.-C. Autrique and F. Magoulès. Numerical analysis of a coupled finite-infinite element method for exterior Helmholtz problems. Journal of Computational Acoustics, 14(1):2143, 2006.

[2] J.-C. Autrique and F. Magoulès. Studies of an infinite element method for acoustical radiation. Applied Mathematical Modelling, 30(7):641-655, 2006.

[3] J.-C. Autrique and F. Magoulès. Analysis of a conjugated infinite element method for acoustic scattering. Computers and Structures, 85(9):518-525, 2007.

[4] J. M. Bahi, R. Couturier, and L. Z. Khodja. Parallel gmres implementation for solving sparse linear systems on gpu clusters. In Proceedings of the 19th High Performance Computing Symposia, pages 12-19, San Diego, CA, USA, 2011. Society for Computer Simulation International.

[5] N. Bell and M. Garland. Efficient sparse matrix-vector multiplication on CUDA. Nvidia Technical Report NVR-2008-004, Nvidia Corporation, 2008.

[6] N. Bell and M. Garland. Implementing sparse matrix-vector multiplication on throughput-oriented processors. In Proceedings of the Conference on High Performance Computing Networking, Storage and Analysis (SC'09), pages 1-11, New York, NY, USA, 2009. ACM.

[7] N. Bell and M. Garland. Library cusp website, 2010. Available on line at: http: //cusplibrary.github.io/ (accessed on December 23, 2021). 
[8] X.-C. Cai, M. A. Casarin, F. W. E. Jr, and O. B. Widlund. Overlapping schwarz algorithms for solving helmholtz's equation. In Domain decomposition methods, 10 (Boulder, CO, 1997), page 391-399. Amer. Math. Soc., Providence, RI, 1998.

[9] A. F. Camargos, V. C. Silva, J.-M. Guichon, and G. Meunier. Iterative solution on gpu of linear systems arising from the a-v edge-fea of time-harmonic electromagnetic phenomena. In Parallel, Distributed and Network-Based Processing (PDP), 2014 22nd Euromicro International Conference on, pages 365-371, Feb 2014.

[10] A.-K. Cheik Ahamed and F. Magoulès. Fast sparse matrix-vector multiplication on graphics processing unit for finite element analysis. In IEEE 14th International Conference on High Performance Computing and Communication (HPCC), pages 1307-1314. IEEE Computer Society, 2012.

[11] A.-K. Cheik Ahamed and F. Magoulès. Iterative methods for sparse linear systems on graphics processing unit. In IEEE 14th International Conference on High Performance Computing and Communication (HPCC), pages 836-842. IEEE Computer Society, june 2012.

[12] A.-K. Cheik Ahamed and F. Magoulès. Iterative Krylov methods for gravity problems on graphics processing unit. In IEEE 12th International Symposium on Distributed Computing and Applications to Business, Engineering Science (DCABES), pages 1620. IEEE Computer Society, 2013.

[13] A.-K. Cheik Ahamed and F. Magoulès. Schwarz method with two-sided transmission conditions for the gravity equations on graphics processing unit. In IEEE 12th International Symposium on Distributed Computing and Applications to Business, Engineering Science (DCABES), pages 105-109. IEEE Computer Society, 2013.

[14] A.-K. Cheik Ahamed and F. Magoulès. A stochastic-based optimized Schwarz method for the gravimetry equations on GPU clusters. In Domain Decomposition Methods in Science and Engineering XXI. Springer, 2014.

[15] P. Chevalier and F. Nataf. Symmetrized method with optimized second-order conditions for the Helmholtz equation. In Domain decomposition methods, 10 (Boulder, CO, 1997), pages 400-407. Amer. Math. Soc., Providence, RI, 1998.

[16] M. M. Dehnavi, D. M. Fernandez, and D. Giannacopoulos. Finite-element sparse matrix vector multiplication on graphic processing units. IEEE, 2010.

[17] C. Farhat and F.-X. Roux. A method of finite element tearing and interconnecting and its parallel solution algorithm. International Journal for Numerical Methods in Engineering, 32(6):1205-1227, 1991.

[18] M. Gander, L. Halpern, F. Magoulès, and F.-X. Roux. Analysis of patch substructuring methods. International Journal of Applied Mathematics and Computer Science, 17(3):395-402, 2007.

[19] M. Gander, F. Magoulès, and F. Nataf. Optimized Schwarz methods without overlap for the Helmholtz equation. SIAM Journal on Scientific Computing, 24(1):38-60, 2002.

[20] M. J. Gander, L. Halpern, and F. Nataf. Optimized Schwarz methods. In T. Chan, T. Kako, H. Kawarada, and O. Pironneau, editors, Twelfth International Conference on Domain Decomposition Methods, Chiba, Japan, pages 15-28, Bergen, 2001. Domain Decomposition Press.

[21] I. Harari and F. Magoulès. Numerical investigations of stabilized finite element computations for acoustics. Wave Motion, 39(4):339-349, 2004. 
[22] H. Knibbe, C. W. Oosterlee, and C. Vuik. Gpu implementation of a helmholtz krylov solver preconditioned by a shifted laplace multigrid method. J. Computational Applied Mathematics, 236(3):281-293, 2011.

[23] M. Kreutzer, G. Hager, G. Wellein, H. Fehske, A. Basermann, and A. R. Bishop. Sparse matrix-vector multiplication on gpgpu clusters: A new storage format and a scalable implementation. CoRR, abs/1112.5588, 2011.

[24] R. Li and Y. Saad. GPU-accelerated preconditioned iterative linear solvers, 2010.

[25] P.-L. Lions. On the Schwarz alternating method. I. In R. Glowinski, G. H. Golub, G. A. Meurant, and J. Périaux, editors, First International Symposium on Domain Decomposition Methods for Partial Differential Equations, pages 1-42, Philadelphia, PA, 1988. SIAM.

[26] P.-L. Lions. On the Schwarz alternating method. II. In T. Chan, R. Glowinski, J. Périaux, and O. Widlund, editors, Domain Decomposition Methods, pages 47-70, Philadelphia, PA, 1989. SIAM.

[27] P.-L. Lions. On the Schwarz alternating method. III: a variant for nonoverlapping subdomains. In T. F. Chan, R. Glowinski, J. Périaux, and O. Widlund, editors, Third International Symposium on Domain Decomposition Methods for Partial Differential Equations, held in Houston, Texas, March 20-22, 1989, Philadelphia, PA, 1990. SIAM.

[28] Y. Maday and F. Magoulès. Non-overlapping additive Schwarz methods tuned to highly heterogeneous media. Comptes Rendus à l'Académie des Sciences, 341(11):701-705, 2005.

[29] Y. Maday and F. Magoulès. Absorbing interface conditions for domain decomposition methods: a general presentation. Computer Methods in Applied Mechanics and Engineering, 195(29-32):3880-3900, 2006.

[30] Y. Maday and F. Magoulès. Improved ad hoc interface conditions for Schwarz solution procedure tuned to highly heterogeneous media. Applied Mathematical Modelling, 30(8):731-743, 2006.

[31] Y. Maday and F. Magoulès. Optimized Schwarz methods without overlap for highly heterogeneous media. Computer Methods in Applied Mechanics and Engineering, 196(8):1541-1553, 2007.

[32] F. Magoulès, P. Iványi, and B. Topping. Convergence analysis of Schwarz methods without overlap for the Helmholtz equation. Computers and Structures, 82(22):18351847, 2004.

[33] F. Magoulès, P. Iványi, and B. Topping. Non-overlapping Schwarz methods with optimized transmission conditions for the Helmholtz equation. Computer Methods in Applied Mechanics and Engineering, 193(45-47):4797-4818, 2004.

[34] F. Magoulès, K. Meerbergen, and J.-P. Coyette. Application of a domain decomposition method with Lagrange multipliers to acoustic problems arising from the automotive industry. Journal of Computational Acoustics, 8(3):503-521, 2000.

[35] F. Magoulès and R. Putanowicz. Optimal convergence of non-overlapping Schwarz methods for the Helmholtz equation. Journal of Computational Acoustics, 13(3):525$545,2005$.

[36] F. Magoulès and F.-X. Roux. Lagrangian formulation of domain decomposition methods: a unified theory. Applied Mathematical Modelling, 30(7):593-615, 2006. 
[37] F. Magoulès, F.-X. Roux, and S. Salmon. Optimal discrete transmission conditions for a non-overlapping domain decomposition method for the Helmholtz equation. SIAM Journal on Scientific Computing, 25(5):1497-1515, 2004.

[38] F. Magoulès, F.-X. Roux, and L. Series. Algebraic way to derive absorbing boundary conditions for the Helmholtz equation. Journal of Computational Acoustics, 13(3):433$454,2005$.

[39] F. Magoulès, F.-X. Roux, and L. Series. Algebraic approximation of Dirichlet-toNeumann maps for the equations of linear elasticity. Computer Methods in Applied Mechanics and Engineering, 195(29-32):3742-3759, 2006.

[40] F. Magoulès, F.-X. Roux, and L. Series. Algebraic Dirichlet-to-Neumann mapping for linear elasticity problems with extreme contrasts in the coefficients. Applied Mathematical Modelling, 30(8):702-713, 2006.

[41] F. Magoulès, F.-X. Roux, and L. Series. Algebraic approach to absorbing boundary conditions for the Helmholtz equation. International Journal of Computer Mathematics, 84(2):231-240, 2007.

[42] K. K. Matam and K. Kothapalli. Accelerating sparse matrix vector multiplication in iterative methods using GPU. In G. R. Gao and Y.-C. Tseng, editors, ICPP, pages 612-621. IEEE, 2011.

[43] Nvidia Corporation. Nvidia - library cublas. Available on line at: http://www.nvidia. com/object/cuda_home_new.html (accessed on December 23, 2021).

[44] Nvidia Corporation. CUDA Toolkit 4.0, CUSPARSE Library, 2011. Available on line at: http://developer.nvidia.com/cuda-toolkit-40 (accessed on December 23, 2021).

[45] Nvidia Corporation. CUDA Toolkit Reference MANUAL, 4.0 edition, 2011. Available on line at: http://developer.nvidia.com/cuda-toolkit-40 (accessed on December $23,2021)$.

[46] A. Quarteroni and A. Valli. Domain Decomposition Methods for Partial Differential Equations. Oxford University Press, Oxford, UK, 1999.

[47] F.-X. Roux, F. Magoulès, L. Series, and Y. Boubendir. Approximation of optimal interface boundary conditions for two-Lagrange multiplier FETI method. In R. K. et al, editor, Proceedings of the 15th Int. Conf. on Domain Decomposition Methods, Berlin, Germany, Jul.21-15, 2003, Lecture Notes in Computational Science and Engineering (LNCSE). Springer-Verlag, Haidelberg, 2005.

[48] Y. Saad. Iterative Methods for Sparse Linear Systems. Society for Industrial and Applied Mathematics, Philadelphia, PA, USA, 2nd edition, 2003.

[49] B. Smith, P. Bjorstad, and W. Gropp. Domain Decomposition: Parallel Multilevel Methods for Elliptic Partial Differential Equations. Cambridge University Press, UK, 1996.

[50] A. Toselli and O. Widlund. Domain decomposition methods. Computational Mathematics, 34, 2004.

[51] A. H. E. Zein and A. P. Rendell. From sparse matrix to optimal gpu cuda sparse matrix vector product implementation. In CCGRID, pages 808-813. IEEE, 2010.

[52] A. H. E. Zein and A. P. Rendell. Generating optimal CUDA sparse matrix-vector product implementations for evolving GPU hardware. Concurrency and Computation: Practice and Experience, 24(1):3-13, 2012. 